\title{
Effect of Pump Selection on Fuel Economy in a Dual Clutch Transmission Vehicle
}

\author{
Rahul Ahlawat, Hosam K. Fathy, Chengyun Guo, Byungchan Lee, Jeffrey L. Stein and Dohoy Jung
}

\begin{abstract}
Positive displacement pumps are used in automotive transmissions to provide pressurized fluid to various hydraulic components in the transmission and also lubricate the mechanical components. The output flow of these pumps increases with speed, almost linearly, but the flow requirements often saturate at higher speeds resulting in the excess flow draining back to the sump. This represents a parasitic loss in the transmission leading to a loss in fuel economy. To overcome this issue, variable displacement pumps have been used in the transmission, where the output flow is reduced by controlling the displacement of the pump. The use of these pumps in automatic transmissions, has resulted in better fuel economy as compared to some types of fixed displacement pumps. However, the literature does not fully explore the benefits of variable displacement pumps to a specific type of transmission namely, dual-clutch transmission, that has different pressure \& flow requirements than an epicyclic gear-train. This paper presents an analysis on the effect of pump selection on fuel economy in a five speed dual clutch transmission of a commercial vehicle. Models of the engine, transmission \& vehicle are developed along with the models of two different types of pumps: a fixed displacement gerotor pump and a variable displacement vane pump. The models are then parameterized using experimental data and the fuel economy of the vehicle is simulated on a standard driving cycle. The results suggest that the fuel economy benefit obtained by the use of the variable displacement pump in dual clutch transmissions is comparable to that of automatic transmissions.
\end{abstract}

\section{INTRODUCTION}

Transmission pumps convert the mechanical energy from the engine into hydraulic energy, by delivering the fluid from a low pressure reservoir to a high pressure line. This pressurized fluid is used in the transmission by the clutch packs, bands, regulating valves \& the torque converter. It also provides lubrication to the mechanical components in the transmission reducing their wear, and provides a cooling effect by transferring heat away from these components. Positive displacement pumps are popular in automotive transmissions due to their relatively higher efficiency over a wide range of operating conditions [1]. The flow produced by these pumps increases with the speed of engine, often exceeding the flow requirements at higher speeds. The excess flow is typically drained back to the transmission sump, and therefore the energy used by the pump for generating this excess flow is not converted into useful work. This

Address all correspondence to Rahul Ahlawat (ahlawatr@umich.edu). Rahul Ahlawat and Byungchan Lee are Ph.D students at the University of Michigan, Ann Arbor. Dr. Hosam K. Fathy, and Prof. Jeffrey L. Stein are with the Department of Mechanical Engineering at the University of Michigan, Ann Arbor. Dr. Dohoy Jung is with the Department of Mechanical Engineering at the University of Michigan, Dearborn. Chengyun Guo is with BorgWarner Inc., Auburn Hills, MI. energy is dissipated as heat, increasing the fluid temperature, and reducing its viscosity, thereby degrading the cooling and lubricating performance of the pump [2]. Hence, this excess flow produced by the pump corresponds to a parasitic loss in the transmission, and it increases significantly with the engine speed. Other parasitic losses in the transmission are largely attributed to windage \& churning of the gears, and friction losses in the bearings [3]. The pumping system components used in an automatic transmission can account for up to $20 \%$ of the total parasitic losses in a typical automotive transmission through the EPA city cycle [4]. These parasitic losses can also significantly impact the coastdown performance and fuel economy of the vehicle, and thus the pumping system should be carefully selected to minimize these losses.

Positive displacement pumps can be categorized as either having fixed or variable displacement geometry. Fixed displacement pumps are typically sized to match the needs of the transmission at all speeds, including the minimum pressure requirements at idle. As pump delivery increases with speed, they tend to produce up to 5 to 10 times the required flow at high pressures [3]. Variable displacement pumps attempt to match the variable flow requirements across the operating range of the transmission. One of the popular transmission pump in this class is the variable displacement vane pump (VDP), but it requires stabilization of its control pressure [4]. In a VDP, pressure fluctuations develop due to the feedback interaction between the pump and its regulator, and these oscillations are controlled by proper damping of the regulator spool [5]. This control is vehicle dependent due to the dynamic coupling between the pump and the entire hydraulic circuit [6]. Variable displacement pumps also contain more moving parts and mechanisms to respond to internal forces that develop in their operation [7], and hence, are more expensive (to install \& control) than fixed displacement pumps. In summary, although their use may lead to degradation of hydraulic system stability, they are still popular because they can be operated at part displacement meeting the pumping requirements with fewer parasitic losses. The benefit obtained in such cases is highly dependent upon the pressure and flow requirements of the transmission, which in turn depends upon the transmission design.

This necessitates the use of different pumps for different transmission designs. For example, automatic transmissions in productions use a variety of pumps, including the fixed displacement gerotor, hypocycloidal \& duocentric pumps, and the VDP. Gerotor pumps use trochoidal gear-form and result 
in low leakages, exhibiting improved volumetric efficiency. Hypocycloidal \& duocentric pumps are relatively new to the automotive industry [4], and the improved teeth geometry results in fewer mechanical losses. The use of VDP leads to a better fuel economy in automatic transmissions, at least theoretically, than fixed displacement pumps [3]. The current VDPs used in transmissions typically have built in extra leakage to obtain improved stability, and therefore usually don't fully meet the fuel economy advantage predicted by theory. Continuously variable transmissions (CVTs) require very high pressures, up to $3 \mathrm{MPa}$, and the overall pumping power requirements often represent a very large portion of the overall CVT power losses. This is particularly a problem at low-power conditions typical of the major operating periods during city and highway driving, where CVT efficiency may drop as low as $85 \%$. Hence variable displacement pumps, particularly radial piston pumps, are very popular in CVTs [3] so that the low-end power consumption is improved by matching the power required by the CVT to the power delivered by the pump. Therefore, the fuel economy benefit of using variable displacement pumps is very dependent on transmission design; the benefit being huge for CVTs but less significant for automatic transmissions. Our work examines the impact of pump selection on fuel economy in a dual clutch transmission (DCT) vehicle. This paper develops models to simulate the performance of two different pumps: the gerotor pump, and the VDP, sized for a particular commercial DCT to meet the flow and pressure requirements, and study the variation in fuel economy. Since VDPs are typically more expensive to acquire and control, the simulation results will reflect the magnitude of the fuel economy benefit achieved by their use, if any, and aid the decision in selection of a suitable pump for this transmission. This analysis will also provide insight into the pumping losses in a DCT and the scope for improvements in such systems.

The remainder of the paper is organized such that section 2 briefly describes vehicle system modeling, focussing on models of the engine, transmission, clutch and the pumps. Section 3 presents the method used for identifying parameters from experimental data, and the results of this parameter identification. Section 4 describes the simulation results, highlighting the differences in fuel economy for the use of different pumps in this DCT vehicle, and section 5 draws the conclusions.

\section{VEHICLE SYSTEM MODELING}

This section presents a forward looking model for the commercial vehicle considered in this work. The subsystem models are selected so that they possess sufficient fidelity for fuel economy simulation while being computationally efficient. The driver is modeled as a PI controller commanding the throttle and brake signals. The engine is modeled as a flow source and it drives the transmission pump. The engine speed then undergoes a reduction in the dual clutch transmission. The output torque from the transmission is input to the final drive, and the final drive torque drives a reduced point mass vehicle model. The road load is calculated by accounting for aerodynamic drag, rolling resistance and the road slope. More details of this forward looking model structure are presented in [8].

\section{A. Engine Modeling}

The engine model consists of three main components: a fuel control module, an engine torque map, and a fuel consumption map. The fuel control module is modeled as a PI controller that decides the fuel demand depending on the engine speed, the idle speed set-point, and the driver demand. The amount of fuel injected into the engine is controlled in a manner that is consistent with the driver pedal position except when engine conditions are such that idle controller is necessary. Under these conditions, idle controller engages and overrides the driver pedal position [9]. The engine torque map takes fuel demand, as dictated by the fuel control module, and engine speed as inputs, and outputs the engine torque, $T_{e}$. This engine torque is then used to obtain the engine speed by accounting for the load torque, and the engine inertia. The inputs for the fuel consumption map are also the throttle position and the engine speed, and the output is the amount of fuel consumed in one cylinder per cycle. The output from the fuel consumption map is then integrated to give the fuel consumption over the period of the simulation. Such a representation of engine is popular in the literature for fuel economy simulations and has been adopted by many researchers including in [10].

\section{B. Transmission Modeling}

Fig. 1 shows the schematic of the basic components of a typical dual-clutch transmission (DCT) and the final drive of the concerned vehicle. This DCT's structure is similar to that of a typical manual transmission as it uses lay-shaft design, but it achieves power-shift by the use of two clutches. As compared to a conventional automatic transmission, it lacks a torque converter and contains only two clutches, that help the vehicle in launch and control the operation of gears. The odd and even gears are mounted on different shafts, and the power transmission through these shafts is controlled by these clutches. The gear shifting is achieved by the use of hydraulically actuated synchronizers. The transmission ECU incorporates the shift map and also a preselection algorithm that anticipates the next gear of operation. When a new gear is anticipated based on the driver demand, the synchronizer engages this gear, while the power still continues to flow through the current gear-shaft. When the gear shift command is given, the off-going clutch is released and the on-coming clutch is engaged, resulting in a clutch to clutch shift \& giving power-shift characteristic similar to that of a conventional automatic transmission. Since this transmission uses fewer clutches and no torque converter, its fuel efficiency is typically higher than that of a traditional epicyclic automatic transmission.

In the literature, models of this transmission have been developed to various levels of fidelity, depending upon the intended application. Zhang et al. developed the model of a DCT for simulating its performance on an EPA cycle [11] 


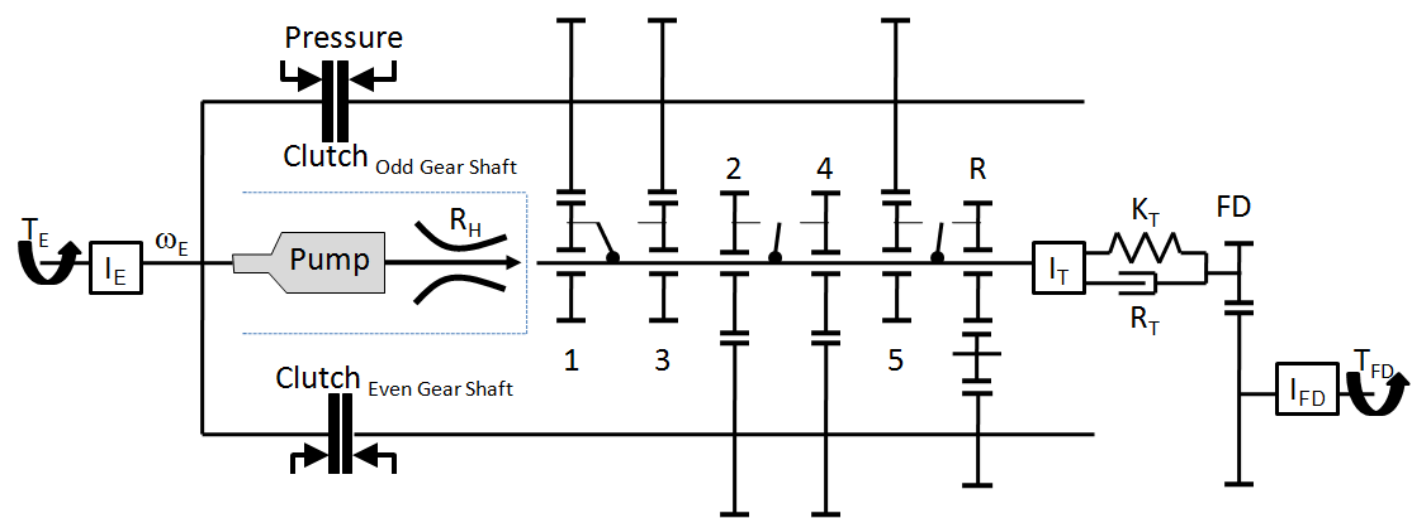

Fig. 1. SChEmatic of The BASIC COMPONENTS OF A TYPICAL DCT \& FINAL DRIVE

whereas Kulkarni et al. developed a dynamic model to study the shift dynamics in a DCT [12]. Here a reduced dynamic lumped parameter model for this transmission is developed by treating gears as modulated transformers with losses, and ignoring the effect of backlash. This representation has been successfully used to model DCTs for performance comparisons [13], and it possesses sufficient fidelity for fuel economy simulations [14]. A two-dimensional shift map is constructed for this vehicle, to obtain the gear of operation based on driver demand \& vehicle speed, such that the engine operates in the region of high torque. This region, for a typical gasoline engine, also overlaps with low bsfc (brake specific fuel consumption) region, giving good fuel economy during vehicle simulation. The shift points are obtained for idle and WOT (wide open throttle) conditions and a linear map is fit to obtain the upshift \& downshift lines. More details on various shift map generation techniques are presented in [10]. In our model, the decision of the shift schedule is delayed by an integration time-step to act as a pre-selection period. During this period the next gear value is considered to be known and its synchronizer is engaged, making the dynamics of the synchronizers unobservable to the power flow. Thus, for the purpose of our simulation, these synchronizers are represented as power flow switches [11], [12]. The hydraulic demand of these synchronizers is accounted in the line-pressure demand of the transmission pump. Windage and churning losses of the transmission are accounted in the model of the hydraulic load.

1) Clutch Modeling: Clutches are used in transmission to transmit torque between two shafts using friction, and hence modeling clutches involves modeling the physics of friction. A comprehensive list of models developed for simulating the dynamics of friction is presented in [15]. These models can be classified as having variable or fixed causality. One of the most fundamental variable causality model is the Coulomb's friction model. In Coulomb's model, clutch friction is modeled as a hybrid system containing two discrete states: a 'slip' state and a 'locked' state. During the 'locked' state, the relative velocity between the friction elements is zero and the friction force is determined by the overall force balance of the system. In 'slip' state, the friction force dictates the slip velocity between the friction elements. Thus, friction acts as a switching element, reversing the causality of the system whenever a transition occurs. Such causality inversions create formulation and computation problems, and can be quite prohibitive [16]. This resulted in the development of fixed causality models of friction, where both the states, 'locked' \& 'slip', maintain the same input-output causality.

Karnopp proposed a fixed causality model that gives friction force as output regardless of whether the friction elements are 'slipping' or 'locked', and is also found to be computationally efficient [17], [18]. Hence, this model is used in the current work for modeling the clutches. In this model, the decision to operate in a discrete state is taken by comparing the clutch slip speed with a small velocity, $\Delta \omega$. Let $\omega_{i n}$ be the input speed to the clutch from the engine, $\omega_{T_{i n}}$ the input speed of the transmission, $T_{c}$ the friction torque transmitted by the clutch and $T_{c a p}$, the clutch capacity. Then, if the slip speed, $\left|\omega_{i n}-\omega_{T_{i n}}\right| \leq \Delta \omega$, and $\left|T_{\text {cap }}\right|>\left|T_{c}\right|$ then the clutch operates in 'locked' state, otherwise in 'slip'state. These conditions specify that at any given time, a clutch cannot transmit more torque than its torque capacity, and if asked to do so while locked, it will start slipping. Also, for the clutch to be 'locked', the slip velocity should be less than $\Delta \omega$, a small velocity used instead of zero velocity to avoid numerical difficulties and prevent hunting between the states. When in the 'locked' state, the angular acceleration remains the same on both sides of the clutch giving an expression for the clutch torque,

$\alpha_{e}=\alpha_{T_{i n}} \Leftrightarrow \frac{T_{e_{n}}-T_{c}}{I_{e}}=\frac{T_{c}-\frac{T_{o}}{N}}{I_{T_{i n}}} \Leftrightarrow T_{c}=\frac{T_{e_{n}}+\frac{T_{o} \cdot I_{e}}{N \cdot I_{T_{i n}}}}{1+\frac{I_{e}}{I_{T_{i n}}}}$

where $\alpha_{e}$ is the angular acceleration of the clutch input shaft, $\alpha_{T_{i n}}$ is the angular acceleration of the clutch output shaft (or input shaft of the transmission), $T_{e_{n}}$ is the net 


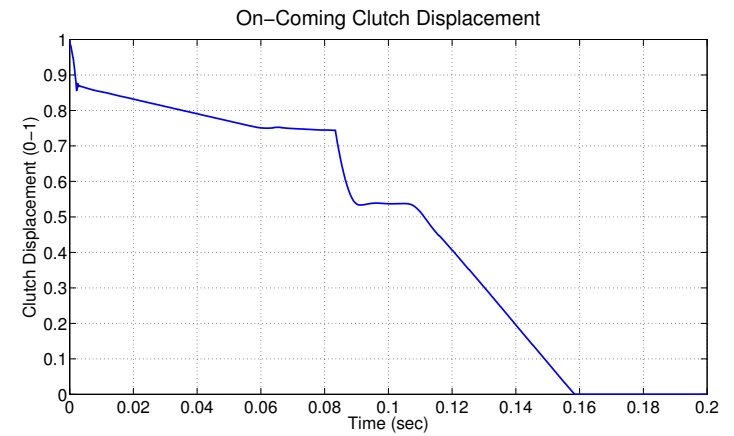

Fig. 2. ON-COMING CLUTCH DISPLACEMENT PROFILE

engine torque after driving the pump, $T_{o}$ is the output torque of the transmission, $N$ is the gear ratio, $I_{e}$ is the inertia of the flywheel and $I_{T_{i n}}$ is the effective inertia of the vehicle as seen on the output shaft of the clutch. If the model is in the 'slip' state then the torque is given by

$$
T_{c}=T_{c a p} \cdot \tanh \left(\frac{\omega_{i n}-\omega_{T_{i n}}}{k}\right)
$$

where $k$ is a numerical constant representing the friction characteristics of the material. The torque clutch capacity, $T_{c a p}$, is itself a function of clutch displacement. A clutch displacement of 0 represents a 'locked' clutch, whereas clutch displacement of 1 represents an 'open' clutch. A separate hydraulic model is constructed to capture the clutch displacement profile, at a constant line pressure, to achieve smooth clutch-to-clutch shifts while reducing the clutch wear and temperature buildup. This is achieved by using a feedback from the off-going clutch pressure to the on-coming clutch pressure, so that tie-up and flare are avoided while maintaining permissible slip speeds and shift-times [19]. This displacement profile is then applied as to the clutches in this model as table function during the gear-shifts. The clutch displacement profile obtained for the on-coming clutch is shown in Fig. 2.

\section{Pump and Hydraulic Load Modeling}

Two different pump models, a fixed displacement gerotor pump and a variable displacement vane pump (VDP), are considered for this work. Both of these pumps have been modeled to various degrees of fidelity in the literature, using both physics based and empirical models. Montero et al. developed a physics based model of gerotor pump capturing chamber to chamber flow fluctuations and used it to study the fluid borne noise [20]. Thompson et al. modeled the VDP as a collection of lumped, compressible volumes separated by sharp edged orifices and studied its control characteristics [6]. Karmel developed various exact and approximate models of the VDP and compared the results to experimental data. He concluded that a simplified model based on mean torque can represent pump dynamics under conditions of lowamplitude eccentricity oscillations, but the model's predictions are invariably biased. The degree of the bias depends on dead-volume effects, and consequently will vary between production units and under different operating conditions [7]. Therefore, most developers rely on experimental data and empirically based representation of losses [21]. Hence steady state models of the pumps predicting mean torque and flow, and representing the losses in a semi-empirical fashion possess sufficient fidelity for fuel economy simulations. Constant temperature and stable regulation of the VDP is assumed for the operation.

Positive-displacement pumps are characterized by a pump constant $D$, that represents flow displacement per unit speed. Variable displacement pumps will require an additional input $x$, the percentage of maximum displacement. The ideal flow produced by a pump, $Q_{i}$, is then given by

$$
Q_{i}=x \cdot D \cdot \omega_{e}
$$

where $\omega_{e}$ is the engine speed, the input speed of the pump. The value of $x$ is taken as 1 for a fixed displacement pump. This mean flow predicted by the model is found to be higher than the actual flow produced in real pumps. The losses are attributed to the entrapment of fluid in dead volumes and leakages along various surfaces in the pump. Cavitation also contributes to the loss but is not the dominating factor [4]. If $Q_{a}$ is the actual flow produced, then we can define the volumetric efficiency of the pump as

$$
\eta_{v} \equiv \frac{Q_{a}}{Q_{i}}
$$

The analytic expression for volumetric efficiency can be obtained by analyzing the physics of the fluid flow. Herzog et al. assumed incompressible fluid undergoing laminar flow and used Poiseuille law to account for the leakage in the pump [2]. This model does not account for the loss due to fluid compression and also loss due to turbulent slip at high speeds. Moreover, leakages are found to be strongly correlated to manufacturing tolerances, wear and distortion under load [4]. Also, the geometry itself does not remain constant under changing operating conditions [22]. This further necessitates the use of a class of semi-empirical loss function models. The modified wilson model [23], [24] is a popular model in this class and is used in this work. This model calculates the volumetric efficiency as

$$
\eta_{v}=1-\frac{C_{s}}{x \cdot S}-\frac{\Delta p}{\beta}-\frac{C_{s t}}{x \cdot \sigma}
$$

where $C_{s}$ is the laminar slip coefficient, $C_{s t}$ is the turbulent slip coefficient, $\Delta p$ is the pressure difference between outlet $\&$ inlet ports and $\beta$ is the bulk modulus of the hydraulic fluid. The dimensionless numbers $S$ and $\sigma$ are defined by

$$
\begin{aligned}
& S \equiv \frac{\mu \cdot \omega}{\Delta p} \\
& \sigma \equiv \frac{\omega \cdot D^{1 / 3}}{\left(2 \cdot \frac{\Delta p}{\rho}\right)^{1 / 2}}
\end{aligned}
$$


where $\rho$ is the density of the fluid and $\mu$ is the dynamic viscosity of the fluid. Using energy balance between the hydraulic and mechanical domains, it can be shown that the torque required to operation ideal frictionless pump, $T_{i}$, is given by

$$
T_{i}=x \cdot \Delta p \cdot D
$$

However due to various mechanical losses taking place in the pump such as friction and viscous drag, a larger torque, $T_{a}$, is required to operate the pump. Thus we can define the mechanical efficiency of the pump, $\eta_{t}$, as

$$
\eta_{t} \equiv \frac{T_{i}}{T_{a}}
$$

The modified wilson model calculates the torque loss due to viscous torque, mechanical friction and hydrodynamic drag, and expresses the mechanical efficiency, $\eta_{t}$, as

$$
\eta_{t}=\frac{1}{1+\frac{C_{v} \cdot S}{x}+\frac{C_{f}}{x}+C_{h} \cdot x^{2} \cdot \sigma^{2}}
$$

where $C_{v}, C_{f}, C_{h}$ are the viscous, frictional and hydrodynamic loss coefficients, respectively. The values of all the coefficients are derived from experimental data in section 3 and validated for both the pump models.

The output flow of the pump passes through hydraulic load. The output of the hydraulic load is the line pressure, and this line pressure acts as input to the pump model. The pumps are sized and regulated so that this flow can constantly produce the desired line pressures. The relationship between the input (pump flow) and the output (line pressure) of the hydraulic load represents a monotonically increasing nonlinear relationship, and hence the hydraulic load is modeled as a non-linear resistor [25]. Its parameters are also identified from the experimental data in the next section.

\section{PARAMETER IDENTIFICATION AND VALIDATION}

In this section we describe the parameter identification of the pump model, and the analysis of the results, followed by the parameter identification of the hydraulic load and effect of temperature variation on these results. To obtain the data for the pump, an experimental setup was used to control the pump input pressure, and speed. In this setup, the input shafts of the pump was spun on a dynamometer at different speeds \& pressures, and also at different displacements (for VDP). The experiments were conducted at a constant temperature, and the torque required and the flow produced by both the pumps were measured. The data was then used to parameterize the coefficients $C_{s}, C_{s t}, C_{v}$, $C_{f}, C_{h} \& \beta$ of the modified wilson model, presented in equations 5-10. Since the effect of the bulk modulus of the fluid, $\beta$, changes with aeration, which in turn changes with the operating pressure [26], $\beta$ was allowed to vary with pressure during the identification process. Similarly, the literature documents the dependence of parameters, $C_{s}$ and $C_{s t}$ on speed [24] and a similar dependence on speed is also exhibited by $C_{v}$. Hence these parameters were allowed to vary with speed. Every experiment was done for a fixed set of $n$ pressures, $\Delta \mathbf{p}=\left\{\delta p_{1}, \delta p_{2}, \ldots, \delta p_{n}\right\}$, and $m$ input speeds, $\Omega=\left\{\omega_{1}, \omega_{2}, \ldots, \omega_{m}\right\}$. Therefore, the parameters that need to be identified from the experimental data are: $\mathbf{C}_{\mathbf{s}}=\left\{C_{s_{1}}, C_{s_{2}}, \ldots, C_{s_{m}}\right\}, \mathbf{C}_{\mathbf{s t}}=\left\{C_{s t_{1}}, C_{s t_{2}}, \ldots, C_{s t_{m}}\right\}$, $\mathbf{C}_{\mathbf{v}}=\left\{C_{v_{1}}, C_{v_{2}}, \ldots, C_{v_{m}}\right\}, \mathbf{B}=\left\{\beta_{1}, \beta_{2}, \ldots, \beta_{n}\right\}, C_{f} \&$ $C_{h}$. Let the flow and torque values obtained experimentally for speed $j \in \Omega$, and pressure $i \in \Delta \mathbf{p}$ be given by $Q_{E_{i j}}$, and $T_{E_{i j}}$, respectively. Then the modified wilson method can be used to get the theoretical values of flow and torque for the same inputs as,

$$
\begin{aligned}
Q_{T_{i j}} & =x D \omega_{j}\left(1-\frac{C_{s_{j}} \Delta p_{i}}{x \mu \omega_{j}}-\frac{\Delta p_{i}}{\beta_{i}}-\frac{\sqrt{2} C_{s t_{j}} \Delta p_{i}^{1 / 2}}{x \omega D^{1 / 3} \rho^{1 / 2}}\right) \\
T_{T_{i j}} & =x D \Delta p_{i}\left(1+\frac{C_{v_{j}} \mu \omega_{j}}{x \Delta p_{i}}+\frac{C_{f}}{x}+\frac{C_{h} \rho x^{2} \omega_{j}^{2} D^{2 / 3}}{2 \Delta p_{i}}\right)
\end{aligned}
$$

The error between the experimental values and theoretical values, $E_{Q_{i j}}$ and $E_{T_{i j}}$ is then calculated as,

$$
\begin{aligned}
E_{Q_{i j}} & =\left|Q_{S_{i j}}-Q_{E_{i j}}\right| \\
E_{T_{i j}} & =\left|T_{S_{i j}}-T_{E_{i j}}\right| \\
\mathbf{E} & =\left\|\mathbf{E}_{\mathbf{Q}}\right\|_{2}+\left\|\mathbf{E}_{\mathbf{T}}\right\|_{2}
\end{aligned}
$$

Since the expressions for the flow and torque errors do not contain any common parameters that need to be identified, $\mathbf{E}$ will be minimum when both $\left\|\mathbf{E}_{\mathbf{Q}}\right\|_{2}$ and $\left\|\mathbf{E}_{\mathbf{T}}\right\|_{2}$ are minimum. Therefore the following optimization problem is formulated to identify the pump parameters:

$$
\min _{\mathbf{C}_{\mathbf{s}}, \mathbf{C}_{\mathbf{s t}}, \mathbf{C}_{\mathbf{v}}, \mathbf{B}, C_{f}, C_{h}} \mathbf{E}
$$

subject to $C_{s_{j}}, C_{s t_{j}}, C_{v_{j}}, C_{f}, C_{h} \geq 0$, and $\beta_{i} \geq \beta_{a}$

where $\beta_{a}$ is the bulk modulus of the air at ambient temperature. The solution gives the optimum parameter values for the model. The parameter identification results give considerable insight into system losses. Fig. 3 shows that the laminar leakage coefficient increases with speed, thereby increasing the overall contribution of laminar slip to total leakage, before abruptly dropping at higher speeds. This happens at a critical speed where the turbulent leakage becomes predominant and the coefficient of turbulent leakage starts increasing, as shown in Fig.4. The coefficient of viscous loss, $C_{v}$, is found to decrease with increasing speed, although the overall viscous drag may still increase due to the linear dependence on velocity in the model. The bulk modulus shows strong dependence on pressure. It is a function of the air trapped in the fluid, and since air is more compressible, gross modulus of the fluid is lower than its theoretical value. As the pressure of the fluid is increased until the critical pressure, the air bubbles dissolve into the fluid and increases the bulk modulus of the oil. However, increasing the pressure above the critical pressure does not result in any noticeable 


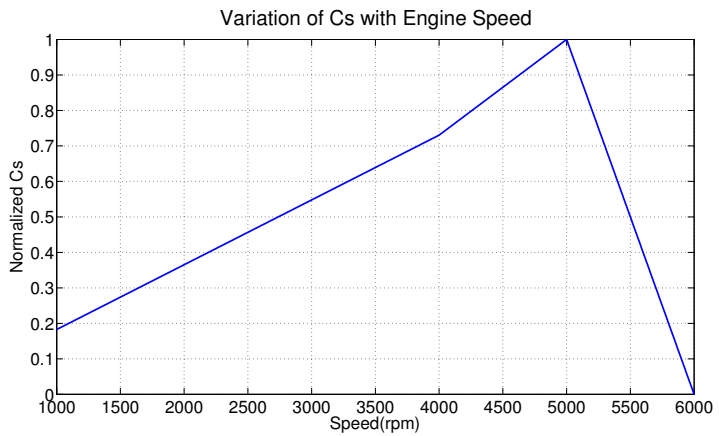

Fig. 3. VARiation of $C_{s}$ With SPEED

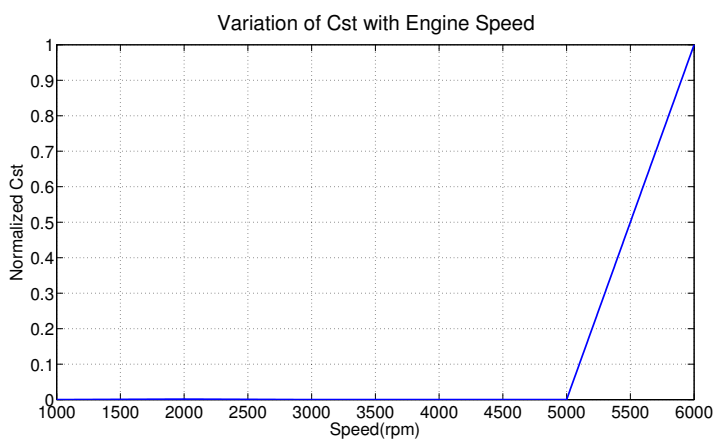

Fig. 4. VARiation of $C_{s t}$ With SPEED

increase in its value [26]. This trend is represented in the identified value of the gross bulk modulus in Fig.6. All the plots have been normalized to protect propriety data.

The parameter identification was carried out using one set of experimental data, and the results were validated against other data sets. For the VDP, identification was done using the data for maximum displacement and validation of the model was done using the data for part displacements. Fig.7 shows the normalized values of pump torques vis-avis the model predictions for maximum displacement in the VDP, and Fig.8 shows the results for $50 \%$ displacement. Similar results were obtained for other displacements as well. All the results indicate good agreement of the model with

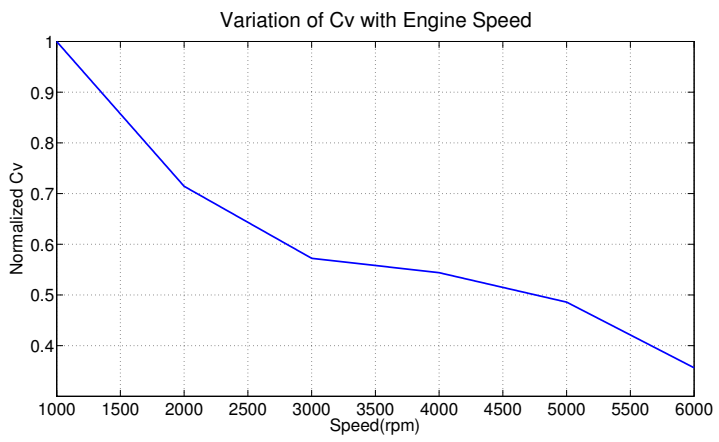

Fig. 5. VARIATION OF $C_{v}$ WITH SPEED

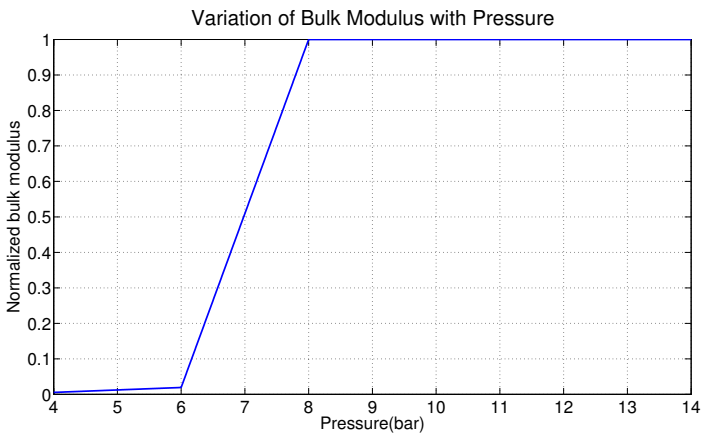

Fig. 6. VARIATION OF $\beta$ WITH PRESSURE

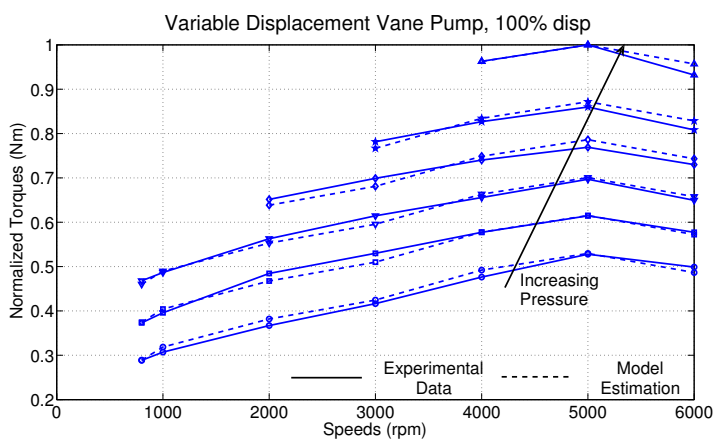

Fig. 7. VARIABLE DisPlacement VANE PUMP: TORQUE DATA FIT

experimental data, giving reasonably small errors, and thus validating our pump model.

The data required for identifying the hydraulic load was obtained by spinning the complete transmission-pump system on a dynamometer and measuring the torque consumed at different speeds and gears. This experimental data, with the appropriate pump model, was then used to derive the pressure-flow data points for the hydraulic load, so that the pump model would consume the same torque at any given speed/gear. These data points were then curve-fitted on a logarithmic curve to obtain physically meaningful resistor model of the hydraulic load. This model accounts for the windage and churning losses taking place in the transmission,

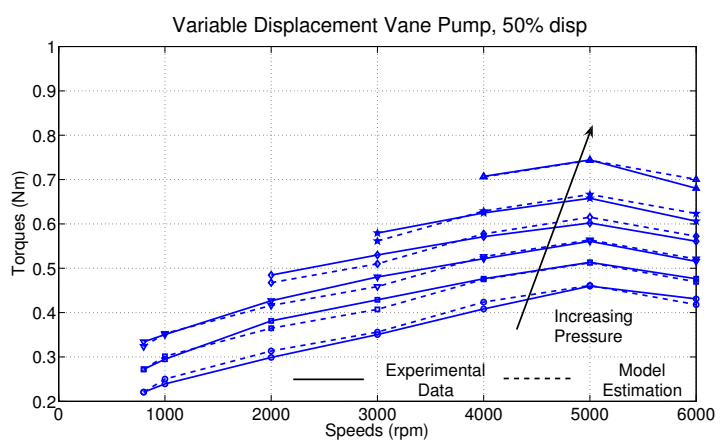

Fig. 8. VARIABLE DISPLACEMENT VANE PUMP: TORQUE FIT VALIDATION 


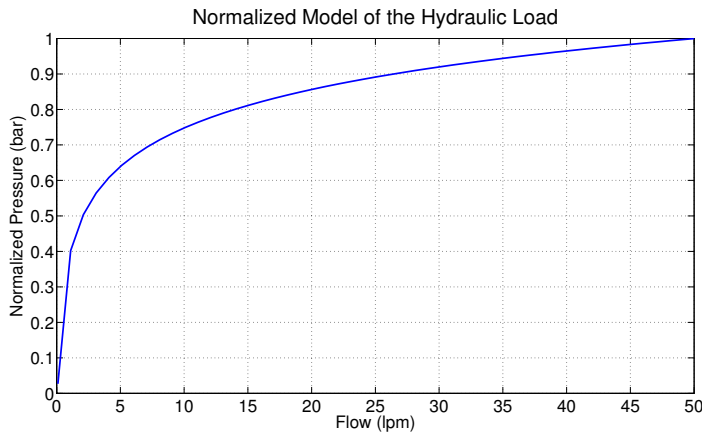

Fig. 9. NORMALIZED MODEL OF THE HYDRAULIC LOAD

and these losses vary significantly with the gear \& lubrication demand of the transmission. Therefore, different curves were obtained for different gear/lubrication operations. A sample normalized hydraulic resistance model is presented in Fig.9.

Experimental data were also obtained by running the tests at different temperatures and parameter identification was carried out for each data set. With increasing temperature, the viscous loss coefficient increased slightly whereas the friction coefficient decreased, resulting in an overall significant increase in mechanical efficiency of the pump. The data also suggests lower volumetric efficiency at higher temperatures, reducing the output pump flow. Also, the hydraulic resistance increases with temperature, producing higher pressures at higher temperatures for the same flow. Thus the overall effect of operating at higher temperatures on pump performance is low torque consumption and slightly lower flow production.

\section{RESUlts AND Discussion}

The models of the engine, driver, transmission, pump and road load were integrated and the simulations were run on the EPA city and highway cycles. The gerotor and the VDP at full displacement produced flows that were significantly higher than the flow requirements for the DCT, especially at higher engine speeds. Treating the fuel economy produced by the gerotor pump as the baseline, the VDP at full displacement produced an improvement of $0.26 \%$ on the city cycle and $0.24 \%$ on the highway cycle. When the displacement of the VDP was controlled to match the desired flow, improvements of $1.45 \%$ and $2.06 \%$ were produced on the city and highway cycles respectively. The flow produced was still higher than the required flow at certain speeds, but operating at part displacements, the parasitic losses were significantly reduced. This potentially represents a substantial efficiency improvement in the DCT. Running the same simulation at different temperatures indicated an increase in fuel economy for all the pumps since the lower flow produced still exceeds the desired flow requirements but the pumping losses in the transmission are reduced at higher temperatures.

The results suggest that the advantages of variable displacement pumps in this typical dual clutch transmission are comparable to that of an automatic transmission, in spite of being structurally different with fewer clutches and no torque converter. This benefit over the gerotor pump, however, comes at a higher cost of the pumping system and is not fully achievable in practice. This is due to the instability associated with the regulation of the control pressure, a common practice in the industry is to bleed the fluid out of the regulation chamber. This degrades its overall system efficiency and hence this fuel economy benefit might be reduced. Improving the margin of stability in the VDP by incorporating design \& control changes, would help in receiving the most benefit out of this system.

\section{Summary AND CONCLUSION}

In summary, this paper presented the development of a dual clutch transmission model and a steady state pump model in detail and the latter was then parameterized for gerotor and VDP using experimental data. These models were integrated with the rest of the vehicle sub-system models and were used for fuel economy simulations on FTP cycle where variable displacement pump showed substantial improvements in the fuel economy. This result suggests that the pumping losses in a DCT can be reduced, at least theoretically, by the use of a VDP. The benefit is also dependent upon the operating range of the engine, and increases as more time is spent at higher speeds. Increasing temperature also results in a better fuel economy.

\section{ACKNOWLEDGEMENT}

We would like to acknowledge Borg-Warner Inc. for providing us with the data used to develop the models, and the technical and financial support for this project.

\section{REFERENCES}

[1] D. B. Parker, "Positive displacement pumps-performance and application," in Proceedings of the $11^{\text {th }}$ international pump user symposium, Texas A\&M University, College Station, Texas, 1994.

[2] S. N. Herzog and D. Placek, "Predicting the pump efficiency of hydraulic fluids to maximize system performance," in International Off-Highway and Powerplant Congress. Society of Automotive Engineers, 2002.

[3] M. Kluger and D. M. Long, "An overview of current automatic, manual and continuously variable transmission efficiencies and their projected future improvements," in International Congress and Exposition, Detroit, MI. Society of Automotive Engineers, 1999.

[4] M. A. Kluger, D. R. Fussner, and B. Roethler, "A performance comparison of various automatic transmission pumping systems," in International Congress and Exposition, Detroit, Michigan. Society of Automotive Engineers, 1996.

[5] A. M. Karmel, "Stability and regulation of a variable-displacement vane-pump," Transactions of the ASME.Journal of Dynamic Systems, Measurement and Control, vol. 110, no. 2, pp. 203-9, 061988.

[6] D. F. Thompson and G. G. Kremer, "Quantitative feedback design for a variable-displacement hydraulic vane pump," Optimal Control Applications and Methods, vol. 19, no. 2, pp. 63-92, 031998.

[7] A. M. Karmel, "Modeling and analysis of the dynamics of a variabledisplacement vane-pump with a pivoting cam," Transactions of the ASME Journal of Dynamic Systems, Measurement and Control, vol. 110, no. 2, pp. 197-202, 061988.

[8] Z. Filipi, H. Fathy, J. Hagena, A. Knafl, R. Ahlawat, J. Liu, D. Jung, D. Assanis, H. Peng, and J. Stein, "Engine-in-the-loop testing for evaluating hybrid propulsion concepts and transient emissions hmmwv case study," SAE Transactions, Journal of Commercial Vehicles, vol. 115,2006 
[9] D. Assanis, W. Bryzik, N. Chalhoub, Z. Filipi, N. Henein, D. Jung, X. Liu, L. Louca, J. Moskwa, S. Munns, J. Overholt, P. Papalambros, S. Riley, Z. Rubin, P. Sendur, J. Stein, and G. Zhang, "Integration and use of diesel engine, driveline and vehicle dynamics models for heavy duty truck simulation," in International Congress and Exposition, Detroit, MI. Society of Automotive Engineers, 1999.

[10] D. Kim, H. Peng, S. Bai, and J. M. Maguire, "Control of integrated powertrain with electronic throttle and automatic transmission," IEEE Transactions on Control Systems Technology, vol. 15, no. 3, pp. 474 $-482,2007$.

[11] Y. Zhang, X. Chen, X. Zhang, H. Jiang, and W. Tobler, "Dynamic modeling and simulation of a dual-clutch automated lay-shaft transmission," Journal of Mechanical Design, Transactions Of the ASME, vol. 127, no. 2, pp. 302-307, 2005.

[12] M. Kulkarni, T. Shim, and Y. Zhang, "Shift dynamics and control of dual-clutch transmissions," Mechanism and Machine Theory, vol. 42, no. 2, pp. 168-182, 2007

[13] X. Song, J. Liu, and D. Smedley, "Simulation study of dual clutch transmission for medium-dutytruck applications," in Commercial Vehicle Engineering Congress and Exhibition, Chicago, Illinois. Society of Automotive Engineers, 2005.

[14] H. K. Fathy, R. Ahlawat, and J. L. Stein, "Proper powertrain modeling for engine-in-the-loop simulation," in ASME International Mechanical Engineering Congress and Exposition, vol. 74 DSC, Orlando, FL, Nov 5-11 2005, pp. 1195-1201.

[15] B. Armstrong-Helouvry, P. Dupont, and C. D. Wit, "A survey of models, analysis tools and compensation methods for the control of machines with friction," Automatica, vol. 30, no. 7, pp. 1083-138, 07 1994.

[16] D. Margolis, "Fixed causality slip-stick friction models for use in simulation of non-linear systems," Proceedings of the Institution of Mechanical Engineers.Part I: Journal of Systems and Control Engineering, vol. 219, no. 3, pp. 199-206, 2005.

[17] D. Karnopp, "Computer simulation of stick-slip friction in mechanica dynamic systems," Journal of Dynamic Systems, Measurement and Control, Transactions ASME, vol. 107, no. 1, pp. 100-103, 1985.

[18] J. Deur, J. Asgari, and D. Hrovat, "Modeling of an automotive planetary gear set based on karnopp model for clutch friction," in ASME International Mechanical Engineering Congress, vol. 72. Publ by ASME, Nov 15-21 2003, pp. 903-910.

[19] S. Bai, R. L. Moses, T. Schanz, and M. J. Gormam, "Development of a new clutch-to-clutch shift control technology," in SAE 2002 World Congress, March 4-7, 2002.

[20] P. J. Gamez-Montero and E. Codina, "Flow characteristics of a trochoidal-gear pump using bond graphs and experimental measurement. part 1," Proceedings of the Institution of Mechanical Engineers, Part I (Journal of Systems and Control Engineering), vol. 221, no. 13, pp. 331-46, 052007.

[21] P. H. Sorensen, E. Trostmann, and F. Conrad, "On the loss function in hydrostatic machines," in ASME Winter Annual Meeting. Atlanta, GA, USA: Publ by ASME, Dec 1-6 1991, pp. 1-5.

[22] D. McCandlish and R. Dorey, "Steady state losses in hydrostyatic pumps and motors," $6^{\text {th }}$ International Fluid Power Symposium, pp. 133-144, 1981.

[23] A. Pourmovahed, N. H. Beachley, and F. J. Fronczak, "Modeling of a hydraulic energy regeneration system. part i. analytical treatment," Journal of Dynamic Systems, Measurement and Control, Transactions of the ASME, vol. 114, no. 1, pp. 155-159, 1992.

[24] D. McCandlish and R. E. Dorey, "Mathematical modeling of hydrostatic pumps and motors," Proceedings of the Institution of Mechanical Engineers, Part B: Management and Engineering Manufacture, vol. 198 , no. 10 , pp. $165-174,1984$.

[25] P. Dransfield, Hydraulic control systems-design and analysis of their dynamics. Springer-Verlag, 1981. 1981.

[26] J. Ruan, X. Pei, and F. M. Zhu, "Identification and modeling of electrohydraulic force control of the material test system (mts)," Journal of Physics: Conference Series, vol. 48, no. 1, pp. 1322-6, 2006 